\title{
Thin Film Composite Membranes: Mechanical and Antifouling Properties
}

\author{
Norin Zamiah Kassim Shaari ${ }^{1, *}$, Norazah Abd Rahman ${ }^{1}$, Nurul Aida Sulaiman ${ }^{1}$, Ramlah \\ Mohd Tajuddin ${ }^{2}$ \\ ${ }^{1}$ Faculty of Chemical Engineering, Universiti Teknologi MARA (UITM), 40450 Shah Alam, \\ Selangor Darul Ehsan, Malaysia \\ ${ }^{2}$ Faculty of Civil Engineering, Universiti Teknologi MARA (UITM), 40450 Shah Alam, Selangor \\ Darul Ehsan, Malaysia
}

\begin{abstract}
As compared to membranes produced from pure polymer or pure inorganic materials, a hybrid membrane possesses better mechanical and thermal properties. This paper reported on the effect of incorporating silica nano-precursor (tetraethylorthosilicate) as well as glycerol in the formulation of hybrid membrane on the mechanical properties and antifouling properties of the resultant thin film composite membranes. The mechanical properties were measured in terms of tensile strength, tensile strain and elastic modulus. Whereas for antifouling properties, it was evaluated through the measurements of relative flux decay (RFD) and relative flux recovery (RFR), along with the permeate flux rate, percentage glycerol permeated and $\mathrm{NaCl}$ rejection. Results showed that the presence of silica and glycerol in hybrid membrane's formulation had increased the tensile strength and elongation of the resultant membranes. In addition to that, the incorporation of glycerol has resulted in thin film composite with better antifouling properties as compared to the thin film composite with barrier layer from the pure polymer blend. Based on its performance, the fabricated thin film composite has a great potential to be used as a pathway for crude glycerol purification due to some advantages over the existing process that employ membrane.
\end{abstract}

\section{Introduction}

In the previous paper [1], it was reported that the incorporation of glycerol in the hybrid membrane formulation had increased the thermal stability and porosity of the resultant membrane with better performance in crude glycerol purification process as compared to that without it. In the preparation of hybrid membrane, sol-gel method was employed. The sol gel reaction involves the hydrolysis of nano-precursor such as silica and titania and condensation of the resulting hydroxyl groups to form a nanostructure. The nanometerordered inorganic minerals precipitate in situ regularly in the organic polymer matrix forming hydrogen bond between organic phase and inorganic phase, where this strong interaction between the organic polymer and inorganic mineral may result in a hybrid of

* Corresponding author: norinzamiah@salam.uitm.edu.my 
markedly improved mechanical properties [2]. It is therefore, the purpose of this paper is to observe the degree of improvement on the mechanical properties as well as the effect of glycerol incorporation.

Mechanical property of the pressure-driven membranes is very important characteristic to evaluate the strength of the membranes in term of withstanding the trans-membrane pressure during filtration and cleaning such as back flushing, where the membranes have enough strength and not easily broken. The mechanical properties of a membrane usually estimated in terms of tensile strength, tensile strain and elastic modulus [3]. As for the hybrid membranes, the analysis was performed to evaluate the effect of cross-linking by silica nano precursor as well as the incorporation of glycerol on the mechanical strength. Besides the mechanical properties, this paper will report on the evaluation on antifouling properties of the resultant thin film composite membranes. Membrane separation process always suffers from fouling phenomenon which normally causes declining in flux as well as the rejection. In water treatment process, in order to maintain the flux, the operating pressure is increased that will lead to higher energy demand and product water cost [4].

There are three main types of foulants in the feed solution during membrane separation process such as inorganic (clays and mineral particles), biological (bacteria, fungi) and organic (oils and humics) [3]. Since the application of the thin film composite is for crude glycerol purification, this paper only focused on the organic foulant by using the actual crude glycerol solution with $75 \%$ additional water. The main mechanisms of fouling are adsorption of partially rejected matter within the membrane pores (pore constriction), plugging of the pores by particle which has similar size as the pores (pore blocking) and cake formation where the top of membrane surface is accumulated with the rejected particles [5]. Fouled membranes are traditionally cleaned using a combination of chemical and physical methods. Pore blocking and cake formation are reversible fouling and could be cleaned by physical hydraulic back flushing unlike pore constriction which is irreversible but could be cleaned by aggressive chemical cleaning process [4]. Besides that, the reversible fouling could be reduced by introducing and increased stirring near the membrane surface. Realizing the above negative impacts caused by fouling phenomenon, fouling resistant membranes are highly desirable for membrane separation process. In this study, the dead-end mode filtration was employed that would impose on severe fouling scenario as compared to cross-flow mode where the shearing force could reduce the fouling effect.

\section{Materials and Methods}

\subsection{Materials}

For the preparation of thin film composite membrane, the material were polyvinyl alcohol with degree of hydrolysis of 87-89\% (molecular weight: $85000-124000 \mathrm{~g} / \mathrm{mol}$ ), polysulfone resin pellet (molecular weight: 44,000-53,000 g/mol), polyethylene glycol (molecular weight: $400 \mathrm{~g} / \mathrm{mol}$ ), pure glycerol, tetraethylorthosilicate with $99 \%$ purity as silica nanoprecursor, and hydrochloric acid with $37 \%$ purity as the catalyst. All the chemicals were obtained from Sigma Aldrich, Malaysia. 1-methyl-2-pyrrolidone with purity of 99\% was obtained from Merck, Malaysia and deionized water as solvent. Crude glycerol was obtained from one of the biodiesel manufacturers in Malaysia. 


\subsection{Methods}

\subsubsection{Preparation of micro porous support membrane}

In the preparation of support membrane, phase inversion technique was employed. Polysulfone polymer solution was prepared by dissolving specific amount of polysulfone pellet into 1-methyl-2-pyrrolidone as the solvent to yield $11 \% \mathrm{wt} / \mathrm{wt}$ concentration of polymer solution. The solution was continuously stirred at $60^{\circ} \mathrm{C}$ until homogeneous solution was achieved. Then, the solution was left for several hours at room temperature for the removal of air bubbles. By using the casting machine and by adjusting the thickness to 45-50 $\mu \mathrm{m}$, the polymer solution was cast onto the glass plate. The film was left in the ambient temperature for 30 seconds before it was immersed in the water as the coagulation medium for 1 hour. Then, the film was kept in the large amount of water for 24 hours. The membrane was subsequently cured at $45^{\circ} \mathrm{C}$ in the oven for 1 hour [6] before preparation of the thin film composite. Fig. 1 shows schematic diagram of the preparation of micro porous support membrane.

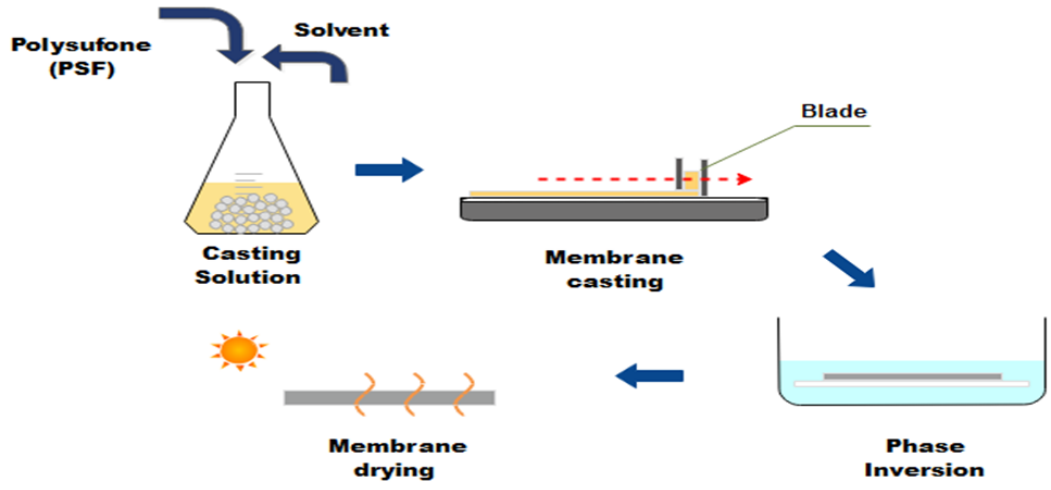

Fig. 1. Formulation of PSF membrane as base membrane (using phase inversion method)

\subsubsection{Preparation of a hybrid membrane}

In the preparation of a hybrid membrane, a sol-gel method was employed. The measured amount of polyvinyl alcohol (PVA) was blended with polyethylene glycol (PEG) with a weight ratio of 9:1 [7]. A measured amount of deionized water as solvent was added to the mixture and the solution were heated at $90^{\circ} \mathrm{C}$ until homogeneous solution was obtained. Glycerol acted as organic additive was added to the solution, which was then stirred at $90^{\circ} \mathrm{C}$ for 15 minutes. The mixture was left to cool down at room temperature. By using sol-gel method, tetraethylorthosilicate (TEOS) as nano precursor at fixed concentration was added at to the solution. Then, $1 \mathrm{ml}$ of hydrochloric acid (37\% purity) was added as the catalyst. The solution was continuously stirred for 10 hours at $30^{\circ} \mathrm{C}$. The composition of PVA, PEG, glycerol and TEOS used in the preparation of the hybrid membrane for this study was shown in Table 1. Four formulations were prepared, which comprise of membrane from pure polymer blend of PVA/PEG, hybrid membranes incorporated with glycerol and TEOS $2.5 \%$ and $4 \%$ respectively and hybrid membrane with TEOS $4 \%$ but without the incorporation of glycerol. 
Table 1. Formulation of membranes

\begin{tabular}{|l|l|l|l|l|}
\hline \multicolumn{1}{|c|}{ Materials } & \multicolumn{4}{c|}{ Formulation } \\
\hline Blended polymer, PVA:PEG (weight ratio) & F1 & F2 & F3 & F4 \\
\hline Glycerol (wt.\% blended polymer) & $9: 1$ & $9: 1$ & $9: 1$ & $9: 1$ \\
\hline TEOS (wt. \% total polymer) & 0 & 10 & 10 & 0 \\
\hline Total polymer + TEOS (wt.\%) & 0 & 2.5 & 4 & 4 \\
\hline Water (wt.\%) & 5 & 5 & 5 & 5 \\
\hline
\end{tabular}

\subsubsection{Preparation of thin film composite (TFC)}

The polysulfone membrane was attached to a glass plate. A specific concentration of a hybrid membrane solution was poured onto the membrane and by using the glass rod, a thin layer of the hybrid membrane solution was coated onto the membrane. The thin film composite membrane was left for 24 hours at room temperature and subsequently cured in the oven at $45^{\circ} \mathrm{C}$ for one hour [6] before characterization and performance evaluation of the membrane. Fig. 2 below show the schematic diagrams on the preparation of hybrid membrane and thin film composite.

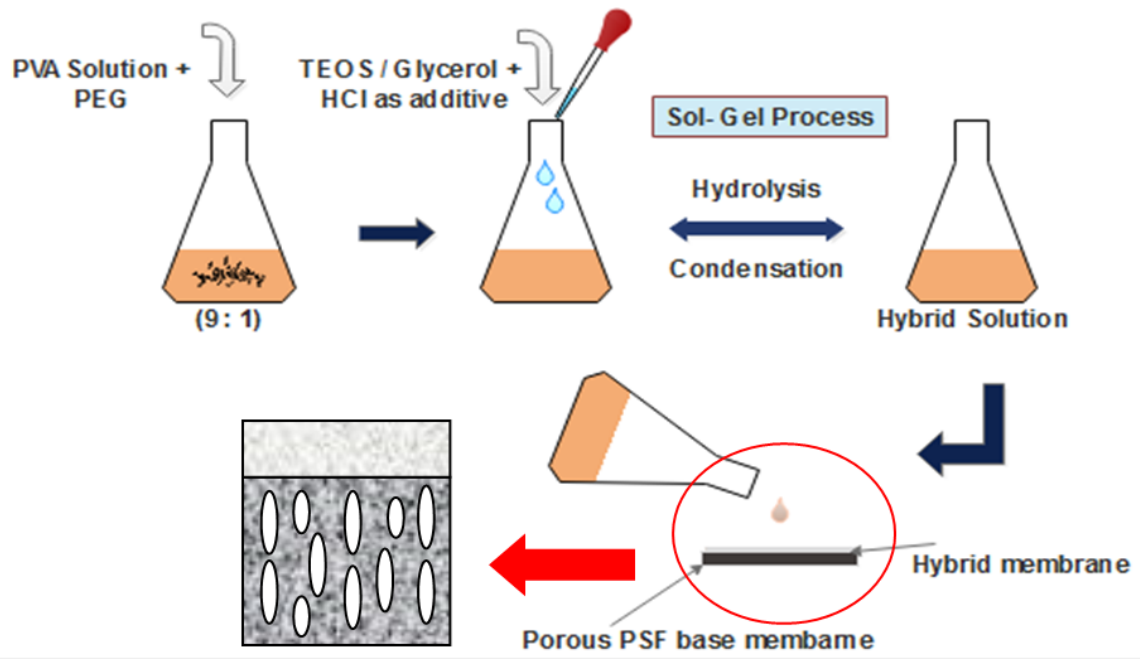

Fig. 2. Preparation of hybrid membrane (using sol-gel method) and thin film composite

\subsection{Characterization}

\subsubsection{Scanning electron microscopy (SEM)}

Cross-sectional of the thin film composite with hybrid membrane (formulation F3) coated on polysulfone membrane from $11 \mathrm{wt}$ \% polymer was observed by using FESEM Supra 40 VP from Carl Zeiss SMT-Nano Technology Systems Division at $5 \mathrm{kV}$ accelerating voltage. Prior to the analysis, the membrane was coated for $50 \mathrm{~s}$ with a 10 -nm-thick layer of gold using a sputter coater (Bal-tec, Model: SCD 005). The test was conducted at the instrumentation laboratory, Faculty of Applied Science, UiTM Shah Alam. 


\subsubsection{Tensile strength measurement}

Mechanical properties of the membranes were measured in the dry state at room temperature using a tensile strength instrument (Model: Instron) with $10 \mathrm{~N}$ load cell and it was conducted in the Instrumentation Laboratory, Faculty of Applied Science, UiTM Shah Alam. Hybrid membranes with a thickness $<100 \mu \mathrm{m}$ were cut into rectangular shape (4 mm width and $100 \mathrm{~mm}$ length) for the measurements of tensile strength, strain and elastic modulus. The test method is according to ASTM D882, which is used for the determination of tensile properties of plastics in the form of thin sheeting with less than 1 $\mathrm{mm}$ thickness. This includes film which has been arbitrarily defined as sheeting having nominal thickness $<0.25 \mathrm{~mm}$. From the stress-strain graph generated from the analysis, the tensile strengths, elongation and elastic modulus were determined. Tensile strength is the maximum stress the film can sustain before it actually fractures or amount of force necessary to pull a material apart. Strain refers to the amount the material will stretch before breaking. Elastic modulus is a measure of the force required to deform the film. It is a measure of the film stiffness. The cross head speed was fixed at $50 \mathrm{~mm} / \mathrm{min}$ and the gauge length was set at $25 \mathrm{~mm}$ [8]. Five samples were tested and the average value was recorded. This test was conducted at instrumentation laboratory, faculty of applied science, UiTM Shah Alam.

\subsubsection{Zeta potential}

The particle size of the synthetic crude glycerol mixtures was measured by using a Zeta Potential Meter with Particle Sizer. Prior to the analysis, the parameters for the analysis and the characteristics of the sample were recorded in the Standard Operating Procedure (SOP) of the software by clicking on 'Start SOP' button. The record was saved. Before conducting the analysis, 'Measure' button followed by 'Start SOP from file' were pressed to retrieve the recorded SOP. Then the sample name was written. Before starting, the crude glycerol solution was prepared according to formulation as shown in Table 2. Then, a test cell was filled with the sample until certain level (the sample height must be below the maximum level). Then the test cell was placed into the closed container. The 'Start' button was pressed and the analysis was executed. The analysis was performed at three cycles. A result was reported in term of size distribution by intensity and an average size of the particles was recorded as $\mathrm{Z}$-average size in $\mathrm{nm}$. The result from the analysis was also shown in Table 2 .

Table 2. Formulation of crude glycerol solution and the particle size

\begin{tabular}{lc}
\hline Compositions & $\begin{array}{c}\text { Crude glycerol } \\
\text { solutions }\end{array}$ \\
\hline Additional water $(\mathrm{wt} . \%)$ & $75 \%$ \\
Crude glycerol $(\mathrm{g})$ & 200 \\
Water $(\mathrm{g})$ & 150 \\
Total solution $(\mathrm{g})$ & 350 \\
\hline Particle size $(\mu \mathrm{m})$ & 5.8 \\
\hline
\end{tabular}

\subsection{Antifouling performance}

The antifouling experiment was performed by using a membrane test machine, which consists of a dead end stirred cell resting on a magnetic stir plate. Crude glycerol solution with 75 wt.\% of additional water was employed as the organic foulant. For each thin film composite membrane, there are three stages involved in the evaluation of anti fouling 
performance. The first stage involved the permeation of distilled water through the thin film composites and the stabilized flux was recorded for 30 minutes $\left(\mathrm{J}_{0}\right)$ [3]. The second stage involved filtration of the crude glycerol solution through the TFC for $2 \mathrm{~h}$ and the flux was recorded as $\mathrm{J}_{\mathrm{P}}$. Then the TFC was cleaned by immersing it into $50 \mathrm{~mL}$ distilled water and being stirred at $200 \mathrm{rpm}$ for $30 \mathrm{~min}$. In the final stage, the pure water flux was recorded again for $30 \mathrm{~min}\left(\mathrm{~J}_{1}\right)$. For each experiment, the feed solution was placed in the feed chamber and was pressurized by using nitrogen gas to permeate through the thin film composite. The flux rate was calculated by dividing the flow rate of the permeate stream with the membrane area. For antifouling performance, two parameters were evaluated such as RFD (relative flux decay) and RFR (relative flux recovery) by the following equations:

$$
\begin{gathered}
\operatorname{RFD}=\left[\left(\mathrm{J}_{0}-\mathrm{J}_{\mathrm{P}}\right) / \mathrm{J}_{0}\right] \times 100 \\
\mathrm{RFR}=\left(\mathrm{J}_{1} / \mathrm{J}_{0}\right) \times 100
\end{gathered}
$$

The compositions of the permeate stream in terms of glycerol content and $\mathrm{NaCl}$ concentration was determined by glycerol content determination method and by using ion meter respectively. Concentration of $\mathrm{NaCl}$ in the feed and permeate was determined by ion meter (Eutech PC 2700 Meter). The salt rejection was calculated by using Equation (3):

$$
R(\%)=\left[1-\frac{C_{p}}{C_{f}}\right] \times 100
$$

where $\mathrm{R}$ is the rejection, $\mathrm{Cf}$ and $\mathrm{Cp}$ are the concentrations of $\mathrm{NaCl}$ in the feed and permeate respectively.

Glycerol content determination was conducted according to standard method, ISO 28791975. The percentage permeation of glycerol (P) was calculated by using equation (4) where $\mathrm{C}_{\mathrm{f}}$ and $\mathrm{C}_{\mathrm{p}}$ are the concentrations of glycerol in the feed and permeate respectively. The process was repeated for three times by using another piece of the TFC and the average values were calculated.

$$
P=\left[\frac{C_{p}}{C_{f}}\right] \times 100
$$

\section{Results and discussion}

Fig. 3 shows a cross sectional view of the TFC, where the presence of the hybrid membrane's layer on the base polysulfone membrane could be observed clearly. Based on the figure, no distinct pores could be observed from the hybrid membrane's layer. Obviously, the thin layer acts as the barrier layer, which dominates the transport resistance of the thin film composite [9] in which the rejection of $\mathrm{NaCl}$ and impurities will occur during the permeation of crude glycerol. 


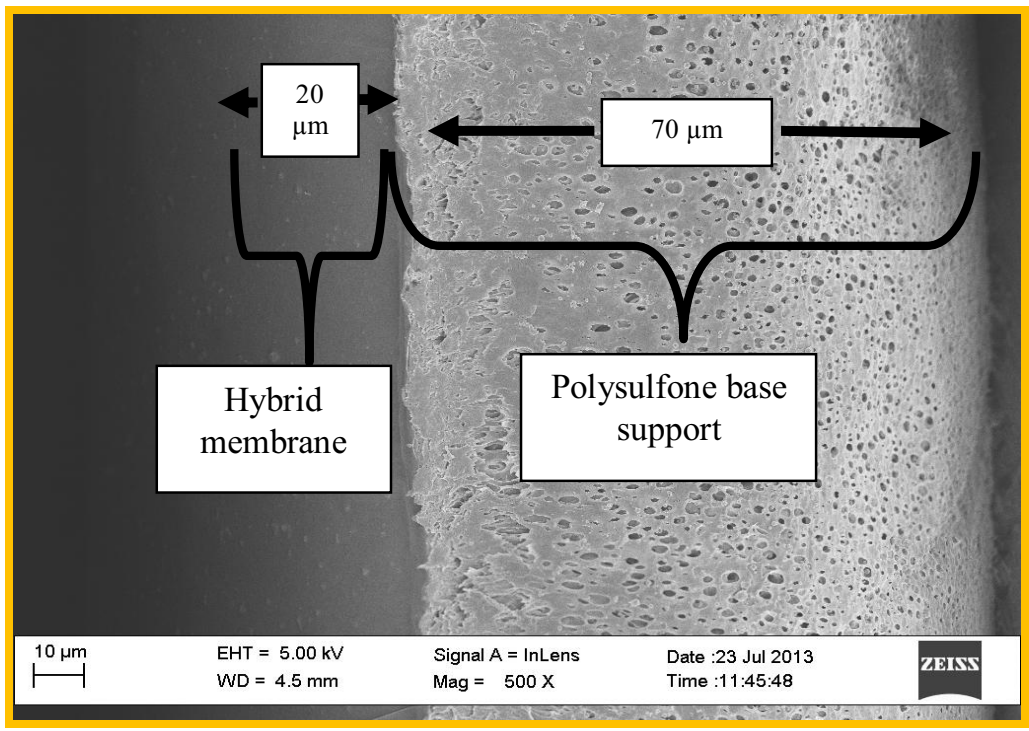

Fig. 3. Cross sectional view of TFC with F3 formulation

Based on Fig. 4, membranes from hybrid formulations, F3 (with glycerol) and F4 (without glycerol) exhibited higher tensile strength and strain as compared to membrane from pure polymer blend (F1). This result had ascertained the claim that cross-linking reaction between organic and inorganic materials resulted in better mechanical properties of the resultant hybrid membrane. From Table 3, it is clearly shown that the incorporation of glycerol in the pure polymer blend (2) has improved the tensile strength and strain of the membrane. As for the hybrid membranes, the mechanical properties of membrane F3, which consists of $10 \mathrm{wt} \%$ glycerol improved significantly as compared to that without glycerol (F5). Based on the figure, it was obvious that a hybrid membrane incorporated with glycerol (F3) has higher tensile strength $(30.22 \mathrm{kPa})$ and strain $(6.20)$ as compared to that without it (F4) where it has tensile strength $15.98 \mathrm{kPa}$ and strain 2.8 . However, based on the elastic modulus values as shown in Table 3 which is determined from the slope of the initial linear portion of the stress vs strain curve, F4 has higher elastic modulus (113.78 $\mathrm{MPa})$ as compared to F3 (48.82 MPa). Elastic modulus represents the stiffness of hybrid membrane, which is resistance to elastic strain [10] and further confirmed through lower elongation that is attributed to the reduction in the ductility of the membrane as stated by Dahe et al. [11]. The needs for good ductility of membrane is important to ensure easy manufacturing of membrane where it will not easily crack and can be easily molded into the required shape. As described by Zubir and Ismail [10], in the production of PTFE (Polytetrafluoroethylene) membrane, the targeted outcomes were membranes with high tensile strength and strain but with low elastic modulus for easy shaping of the membrane into a thin membrane with a uniform thickness.

Generally, the presence of silica in the hybrid membrane could increase the mean distance between polymer chains which causes the increase in stiffness of the polymer structure due to restricted segmental motion [12] that leads to increase in the elastic modulus. The presence of glycerol as the plasticizer has reduced the rigidity structure of the hybrid membrane that is observed through lowering the elastic modulus [13]. Besides that, synergistic effect of glycerol with the cross-linking network portrayed by organic-inorganic materials would substantiate the enhancement in the mechanical properties of the hybrid membrane, which was claimed by Liang et al. [14] in their research on the production of chitosan/polyvinyl alcohol gel films by using cross-linking method. Although the 
incorporation of glycerol has increased the elastic modulus of F3 but the value is marginal and it has been compensated with higher elongation.

Fig. 5 displays the flux change percentage over time from antifouling experiment. As shown in Table 4, all membranes portray high relative flux decay (RFD) due to dealing with high viscosity of feed solution as compared to pure water. However, the flux declining rate for F1 as displayed in Table 4 is higher as compared to other membranes, where membranes from F3 and F4 displaying the lowest RFD. However, all membranes portrayed comparable flux declining rate for filtration on crude glycerol solution. Based on Table 4, after undergoing cleaning process for $30 \mathrm{~min}$, the pure water flux for TFC with barrier layer from F2 and F3 were almost recovered back to the initial values to give relative flux recovery (RFR) $95.51 \%$ and $82.41 \%$ respectively. Whereas for TFC with F1 and F4, lower RFR were measured that shows barrier layer from polymer blend as well as hybrid membrane with the absence of glycerol were easily fouled and it is not a reversible fouling. Higher RFR exhibited by F2 and F3 would justify the enhancement in hydrophilicity of the membrane's surface as a result of glycerol incorporation during the formulation

In term of the performance of all membranes on crude glycerol purification, TFC with hybrid membranes exhibited better permeation of glycerol with sufficient rejection of $\mathrm{NaCl}$ as displayed in Table 4. This situation is caused by the formation of hydrogen bonding between organic phase and inorganic phase during the sol-gel reaction [15], where this property would benefit the crude glycerol purification process as water soluble material could permeate through the membrane. TFC with F3 as the barrier layer shows the best performance in term of rejecting higher percentage of $\mathrm{NaCl}$ by allowing higher percentage of glycerol passing through the TFC.

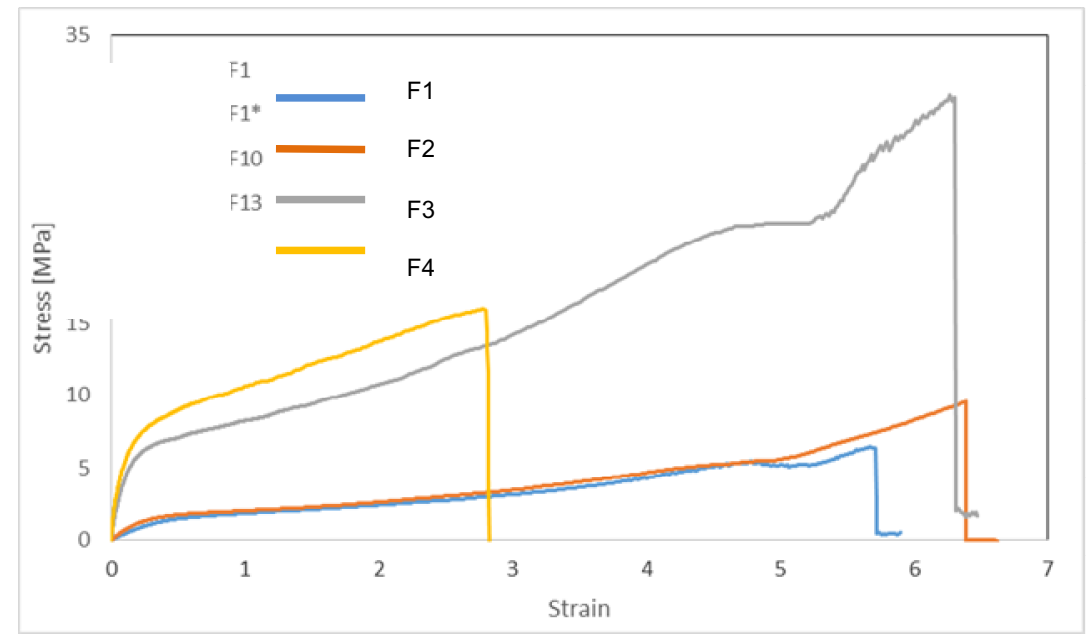

Fig. 4. Stress vs elongation for membranes from various formulations

Table 3. Mechanical properties of various membranes

\begin{tabular}{|c|c|c|c|}
\hline Membrane & $\begin{array}{c}\text { Tensile strength } \\
\text { (MPa) }\end{array}$ & $\begin{array}{c}\text { Break } \\
\text { Strain }\end{array}$ & $\begin{array}{c}\text { Elastic modulus } \\
\text { (MPa) }\end{array}$ \\
\hline F1 & 6.456 & 5.67 & 3.925 \\
\hline F2 & 13.36 & 6.39 & 21.28 \\
\hline F3 & 30.22 & 6.20 & 48.82 \\
\hline F4 & 15.98 & 2.80 & 113.78 \\
\hline
\end{tabular}




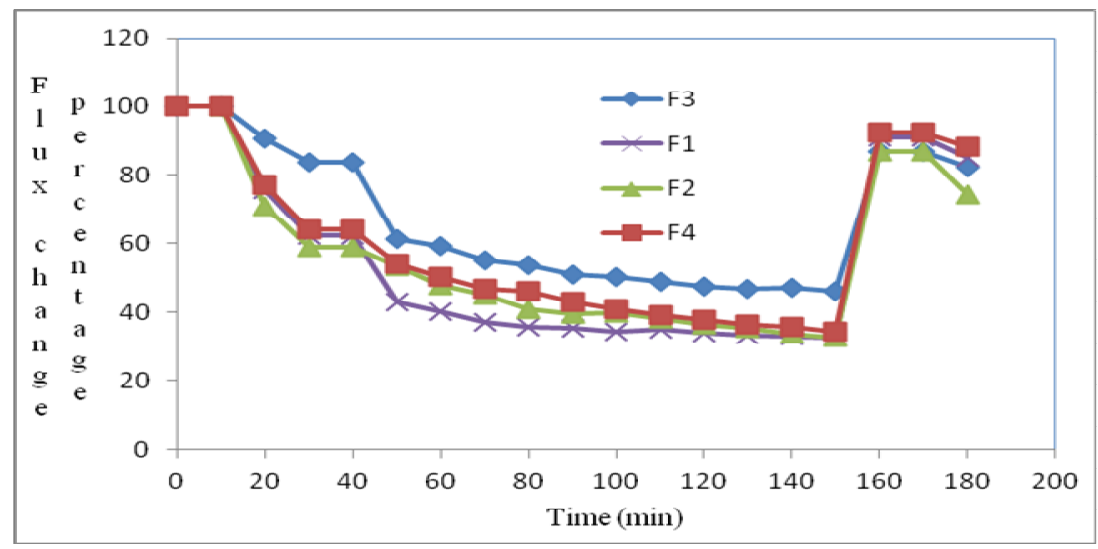

Fig. 5. Variation in the permeate flux of various TFC membranes during filtration of crude glycerol solution

Table 4. Performance of various membranes in term of antifouling behaviour and filtration

\begin{tabular}{|c|c|c|c|c|c|c|c|}
\hline Formulation & $\begin{array}{c}\mathbf{J}_{\mathbf{0}} \\
\left(\mathbf{L} / \mathbf{m}^{2} \text {.day }\right)\end{array}$ & $\begin{array}{c}\mathbf{J}_{\mathbf{P}} \\
\left(\mathbf{L} / \mathbf{m}^{2} \mathbf{. d a y}\right)\end{array}$ & $\begin{array}{c}\mathbf{J}_{\mathbf{1}} \\
\left(\mathbf{L} / \mathbf{m}^{2} . \mathbf{d a y}\right)\end{array}$ & $\begin{array}{c}\mathbf{R F D} \\
\mathbf{( \% )}\end{array}$ & $\begin{array}{c}\mathbf{R F R} \\
\mathbf{( \% )}\end{array}$ & $\begin{array}{c}\text { NaCl } \\
\text { rejection } \\
\mathbf{( \% )}\end{array}$ & $\begin{array}{c}\text { Glycerol } \\
\text { permeated } \\
\mathbf{( \% )}\end{array}$ \\
\hline $\mathrm{F} 1$ & 2484.38 & 52.56 & 1397.12 & 97.88 & 56.24 & 29.8 & 80.02 \\
\hline $\mathrm{F} 2$ & 2033.43 & 55.19 & 1942.13 & 97.28 & 95.51 & 23.01 & 90.74 \\
\hline $\mathrm{F} 3$ & 630.78 & 39.77 & 462.02 & 93.34 & 82.41 & 32.62 & 86.60 \\
\hline $\mathrm{F} 4$ & 1610.14 & 74.56 & 879.77 & 95.37 & 54.64 & 23 & 93.96 \\
\hline
\end{tabular}

\section{Conclusion}

The presence of glycerol in the hybrid membrane formulation had reduced the rigidity structure of the resultant membrane that was observed through the improvement on the tensile strength and elongation with the reduction in the tensile modulus. Furthermore, the enhanced hydrophilicity of the membrane's surface had increased its antifouling behaviour which was displayed through higher relative flux recovery values. Based on its performance, the fabricated thin film composite has a great potential to be used as a pathway for crude glycerol purification due to some advantages over the existing process that employ membrane.

The author would like to thank Ministry of Higher Education for funding the research through Fundamental Research Grant Scheme (FRGS) No. 600-RMI/ST/FRGS 5/3/Fst (100/2010).

\section{References}

[1] K.S. Norin Zamiah and A.R. Norazah, Performance of thin film composite with hybrid membrane as the barrier layer for the separation process of synthesized crude glycerol, Proc. of the 2013 IEEE Business Engineering and Industrial Applications Colloquium, Bayview Hotel, Langkawi, (2013) 
[2] S. Yano, K. Iwata, and K. Kurita, Physical properties and structure of organic-inorganic hybrid materials produced by sol-gel process, Material Science and Engineering., 6(23) , 75-90, (1998)

[3] X.Zhu, H.E. Loo and R. Bai, A novel membrane showing both hydrophilic and oleophobic surface properties and its non-fouling performances for potential water treatment applications, J. of Membrane Science, 436, 47-56, (2013)

[4] E.M.V. Hoek, A.K. Ghosh, X. Huang, M. Liong and J.I. Zink, Physical-chemical properties, separation performance, and fouling resistance of mixed-matrix ultrafiltration membranes, Desalination, 283, 89-99, (2011)

[5] S. Kim, M. Marion, B.H. Jeong and E.M.V. Hoek, Crossflow membrane filtration of interacting nanoparticles suspensions, J. of Membrane Science, 283, 89-99, (2011)

[6] G.J. Francisco, A. Chakma and X. Feng, Membranes comprising of alkanolamines incorporated into poly(vinyl alcohol) matrix for $\mathrm{CO} 2 / \mathrm{N} 2$ separation, J. of Membrane Science, 303, 54-63, (2007)

[7] L.Y.Ye, Q.L.Liu, Q.G. Zhang, A.M. Zhu, and G.B. Zhou, Pervaporation characteristics and structure of Poly(vinyl alcohol) / Poly(ethylene glycol) / Tetraethylorthosilicate hybrid membranes, J. of Applied Polymer Science, 105, 3640-3648, (2007)

[8] N.A. Zubir and A.F. Ismail, Effect of sintering temperature on the morphology and mechanical properties of PTFE membranes as a base substrate for proton exchange membrane, Songklanakarin J. Sci. Technology, 24, 823-831, (2002)

[9] H.J. Li, Y.M. Cao, J.J. Qin, X.M. Jie, T.H. Wang, J.H. Liu and Q.Yuan, Development and characterization of anti-fouling cellulose hollow fiber UF membranes for oil-water separation, J. of Membrane Science, 279, 328-335, (2006)

[10] G.J. Dahe, R.S. Teotia and J. R. Bellare, The role of zeolite nanoparticles additive on morphology, mechanical properties and performance of polysulfone hollow fiber membranes," Chemical Engineering J., 197, 398-406, (2012)

[11] G.J. Dahe, R.S. Teotia and J. R. Bellare, The role of zeolite nanoparticles additive on morphology, mechanical properties and performance of polysulfone hollow fiber membranes, Chemical Engineering J., 197, 398-406,(2012)

[12]M. Sadeghi, M.A. Semsarzadeh and H. Moadel, Enhancement of the gas separation properties of polybenzimidazole (PBI) membrane by incorporation of silica nano particles, J. of Membrane Science, 331, 21-30, (2009)

[13] J.C. Wang, P. Chen, L. Chen, K. Wang, H. Deng, F.Chen, Q. Zhang and Q. Fu, Preparation and properties of poly (vinylidene fluoride) nanocomposites blended with grapheme oxide coated silica hybrids, eXPRESS Polymer Letters, 6(4), 299-307, (2012)

[14] S. Liang, L. Liu, Q. Huang and K.L. Yam, Preparation of single or double-network chitosan/poly(vinyl alcohol) gel films through selectively cross-linking method, Carbohydrate Polymers, 77, 718-724, (2009).

[15] K. Xie, Y. Yu and Y. Shi, Synthesis and characterization of cellulose/silica hybrid materials with chemical crosslinking, Carbohydrate Polymers, 78, 799-805, (2009) 